\title{
openheart Added fructose as a principal driver of non-alcoholic fatty liver disease: a public health crisis
}

\author{
James J DiNicolantonio, ${ }^{1}$ Ashwin M Subramonian, ${ }^{2,3}$ James H O’Keefe ${ }^{1}$
}

To cite: DiNicolantonio JJ, Subramonian AM, O'Keefe JH. Added fructose as a principal driver of non-alcoholic fatty liver disease: a public health crisis. Open Heart 2017;4:e000631. doi:10.1136/ openhrt-2017-000631

Accepted 4 October 2017

\section{CrossMark}

${ }^{1}$ Preventive cardiology, Saint Luke's Mid America Heart Institute, Kansas City, Missouri, USA

${ }^{2}$ Department of medicine, Meenakshi Mission Hospital and Research Centre, Madurai, India ${ }^{3}$ Alumni, Volgograd State Medical University, Volgograd, Russia

\section{Correspondence to} Dr James J DiNicolantonio; jjdinicol@gmail.com

\section{ABSTRACT}

Fatty liver disease affects up to one out of every two adults in the western world. Data from animal and human studies implicate added sugars (eg, sucrose and high-fructose corn syrup) in the development of fatty liver disease and its consequences. Added fructose in particular, as a component of added sugars, may pose the greatest risk for fatty liver disease. Considering that there is no requirement for added sugars in the diet, dietary guidelines should recommend reducing the intake of added sugars to just $5 \%$ of total calories in order to decrease the prevalence of fatty liver disease and its related consequences.

\section{INTRODUCTION}

Fatty liver disease is the presence of hepatic steatosis (fat deposited in the liver). There are two main types of fatty liver disease: non-alcoholic fatty liver disease (NAFLD) and alcoholic fatty liver disease. Alcoholic fatty liver disease, with or without steatohepatitis (inflammation of the liver with concurrent liver fat accumulation), is associated with excessive alcoholic consumption and is typically macrovesicular. NAFLD is a cause of hepatic fat accumulation in the absence of heavy alcohol use. Non-alcoholic fatty liver and non-alcoholic steatohepatitis are the further subdivisions of NAFLD with the absence of inflammation in the former and its presence in the latter. Hepatic triglyceride (TG) concentration (steatosis) exceeding $55 \mathrm{mg} / \mathrm{g}$ liver $(5.5 \%)$ is the earliest stage of NAFLD.

The current tenet suggests central obesity, type 2 diabetes mellitus, dyslipidaemia, male sex and metabolic syndrome are the common major risk factors of NAFLD. ${ }^{2}$ However, recent evidence suggests a positive association between added sugars (particularly added fructose) and NAFLD, metabolic syndrome and weight gain. ${ }^{34}$ NAFLD is seen worldwide and is the most common liver disorder in Western industrialised countries with a prevalence of $10 \%-46 \%$ in the USA, ${ }^{5}$ where there is a high consumption of simple sugars in addition to added fats and oils.

Fructose is a monosaccharide that is sweeter than either glucose or sucrose and is found naturally in fruits and some vegetables. In humans, fructose is almost completely metabolised in the liver with under $1 \%$ directly converted to plasma TGs, $29 \%-54 \%$ of fructose converted in the liver to glucose, about $25 \%$ of fructose is converted to lactate, and $15 \%-18 \%$ is converted to glycogen. ${ }^{6}$ Fructose is poorly absorbed from the gastrointestinal tract because there is a limited amount of intestinal cells that have the glucose transporter type-5 (GLUT-5) transporter through which fructose moves into the cell. However, with increased consumption of fructose there is an upregulation in GLUT-5. ${ }^{7}$ In other words, the more fructose you eat the more you absorb. Hepatic metabolism of fructose stimulates lipogenesis and these events are independent of insulin exertion and phosphofructokinase regulation step. ${ }^{8}$

Due to its primary metabolism in the liver and based on the studies conducted by Dekker et $a l,{ }^{9}$ Chung et $a l^{10}$ and Schwarz et $a l,{ }^{11}$ the development of fatty liver disease from the overconsumption of added fructose should be considered a public health crisis.

\section{MECHANISMS FOR FRUCTOSE CAUSING FATTY LIVER}

De novo lipogenesis (DNL) is one of the mechanisms proposed for how consuming added fructose leads to fatty liver. ${ }^{11}$ Fructose may induce NAFLD by its ability to upregulate DNL and by bypassing the major rate-limiting step of glycolysis at phosphofructokinase.

DNL is a self-promoting process; genes that activate DNL are influenced by sterol regulatory element-binding proteins (SREBP). ${ }^{12} 13$ Fructose independently activates SREBP-1c, which in turn activates DNL. Fructose-induced DNL generates fatty acids that can 
then be incorporated into hepatic TGs or other lipid species. Apolipoprotein B-100 (apoB) is essential for the intracellular assembly of TG into very low-density lipoprotein (VLDL), and apoB degradation is reduced when hepatic lipid is increased. ${ }^{14}$ As a result, hepatic endoplasmic reticulum (ER) stress ${ }^{15}$ occurs due to the accumulation of apoB. The stress in the ER further promotes SREBP-1c activation and thus further contributes to DNL. ${ }^{16}$

Rodríguez-Calvo et $a l^{17}$ demonstrated that fructose feeding has also been shown to induce the activation of carbohydrate-responsive element-binding protein and increase the expression of lipogenic genes such as fatty acid synthase (FAS), acyl coenzyme-A carboxylase and stearoyl coenzyme-A desaturase-1 in the fructose-fed rat.

\section{ANIMAL STUDIES}

A study ${ }^{18}$ on male Sprague-Dawley breeder rats showed similar results. Rats were randomly divided into two groups, consisting of 10 sucrose-fed and a similar number of starch-fed rats followed for 4 months. The sucrose group received a $40 \%$ sucrose diet (protein $18.3 \%$ by weight and $20.2 \% \mathrm{kcal}$; carbohydrate $60.5 \%$ by weight (consisting of $40 \%$ sucrose and $20.5 \%$ starch) and $66.9 \%$ kcal; fat $5.2 \%$ by weight and $12.9 \% \mathrm{kcal}$ ). The control group received an isocaloric diet in which the sucrose was replaced by starch (protein $18.3 \%$ by weight and $20.2 \%$ kcal; carbohydrate $60.5 \%$ by weight and $66.9 \%$ kcal; fat $5.2 \%$ by weight and $12.9 \% \mathrm{kcal})$. The results revealed fatty liver with macrovesicular and microvesicular steatosis. On western blotting, there was increased FAS and ATP citrate lyase (involved in fat synthesis) and decreases in enoyl-CoA hydratase (ECoAH1) (involved in $\beta$ fatty acid oxidation). Hence, the studies ${ }^{19}{ }^{20}$ suggest that sucrose and fructose induce both an increase in fat synthesis and a decrease in fat oxidation.

Ackerman $e t a l^{21}$ performed a study on Sprague-Dawley rats. Forty-nine male Sprague-Dawley rats weighing $200 \pm 20 \mathrm{~g}$ were studied. The rats were randomly divided into five groups. One group continued to be maintained on standard rat chow diet (SRCD) for 5 weeks, whereas the other four groups were given a fructose-enriched diet (FED). The FED contained 20.7\% (per weight basis) protein (as casein), 5\% fat (as lard), 60\% carbohydrates (as fructose), $8 \%$ cellulose, $5 \%$ mineral mix and $1 \%$ vitamin mix, and $50 \mathrm{mg}$ of iron in $1 \mathrm{~kg}$ of diet. The SRCD contains $21.9 \%$ protein, $4.5 \%$ fat, $41 \%$ starch, $5 \%$ sugar, and $3.7 \%$ crude fibre and $120 \mathrm{mg}$ of iron in $1 \mathrm{~kg}$ of diet. Three weeks after the initiation of FED, three out of the four groups were given various additional pharmaceutical interventions for 2 weeks. One group was given amlodipine $(15 \mathrm{mg} / \mathrm{kg} /$ day $)$, another group was given captopril $(90 \mathrm{mg} / \mathrm{kg} /$ day $)$ and the third group was given bezafibrate $(10 \mathrm{mg} / \mathrm{kg} /$ day $)$. The fourth group, the control group, did not receive any pharmaceutical intervention and remained in FED. FED rats developed hepatic macrovesicular and microvesicular fat deposits, with increases in hepatic TGs $(+198 \%)$ and hepatic cholesterol $(+89 \%)$, hypertriglyceridaemia $(+223 \%)$, and hypertension $(+\sim 15 \%$ in blood pressure), but a decrease in hepatic phospholipids $(-36 \%)$, without increases in hepatic iron concentration (HIC). Amlodipine reduced blood pressure $(-18 \%)$ and plasma TGs $(-12 \%)$ but there was no change in hepatic TGs and phospholipid concentrations. Captopril reduced blood pressure $(-24 \%)$, plasma TGs $(-36 \%)$, hepatic TGs $(-51 \%)$ and hepatic macrovesicular fat $(-51 \%)$, but increased HIC $(+23 \%)$, with a borderline increase in hepatic fibrosis. Bezafibrate reduced plasma TGs $(-49 \%)$, hepatic TGs $(-78 \%)$, hepatic macrovesicular fat $(-90 \%)$ and blood pressure $(-11 \%)$. In summary, high-fructose feeding induces fatty liver deposits in rats.

Another study ${ }^{22}$ supports the role of fructose feeding and steatohepatitis. Twenty-four rats at random were divided into three groups ( $\mathrm{n}=8$, each group): the control group $(\mathrm{C})$, which received a purified diet; the high-fructose-fed group (F); and the high-fructose-fed and Thymosin alpha 1 injected group $(\mathrm{F}+\mathrm{T})$. After the experimental period of 10 days, liver lipid peroxidation and antioxidant status, and blood interleukin (IL)-1-beta, IL-2 and IL-6 levels were quantified. The results showed that in comparison with the $\mathrm{C}$ group, the $\mathrm{F}$ group had a higher xanthine oxidase activity and lipid peroxidation, as indicated by concentrations of thiobarbituric acid reactive substances, and lower superoxide dismutase and glutathione peroxidase activities in the liver. In the $\mathrm{F}+\mathrm{T}$ group, these markers were near the values of the control group. In addition, increased IL-1-beta and IL-6 levels were kept at near to normal levels with treatment of Thymosin alpha 1, but not IL-2 levels. In the F group, the most consistent findings in the histological sections of liver tissues were the macrovesicular and microvesicular steatosis. Thymosin alpha 1 treatment protected the majority of the liver cells, while minimal macrovesicular and microvesicular steatosis was observed in the remaining cells. These results show that a high-fructose diet ( $\mathrm{HFr}$ ) in rats leads to hepatic steatosis and a defect in the free radical defence system, and that treatment with Thymosin alpha 1 may improve these biochemical and morphologic changes in the fructose-fed rat livers.

A very recent study ${ }^{23}$ on 27 cynomolgus monkeys (Macaca fascicularis) over 7 years demonstrated that high-carbohydrate diets alone can generate both liver fat and fibrosis. The monkeys were fed diets with two diets: a control diet (CTL, $n=10)$ or an HFr diet $(n=17)$. The CTL diet was high in carbohydrates (69\% of calories), with less than $3 \%$ glucose and $0.5 \%$ fructose supplying the calories. The HFr diet was equivalent in the percentage of calories supplied by carbohydrates; however, $24 \%$ of the total caloric intake was supplied from fructose. The results showed that fructose-fed monkeys were heavier than control animals $(3.03 \pm 0.09 \mathrm{~kg}$ vs $5.42 \pm 0.83 \mathrm{~kg}$; $\mathrm{p}=0.04$ ). The HFr diet also increased the number of lipid droplets, that is, steatosis score $(\mathrm{p}<0.001)$, although the droplets were of smaller average diameter $(\mathrm{p}=0.002)$. 
The diet high in fructose also significantly increased liver collagen content. Collagen in hepatic tissue was increased with fructose consumption when measured as either a percentage of the entire histological section $(\mathrm{p}=0.03)$ or the liver parenchyma that was distributed between the portal triads $(p=0.007)$. These pathological features resembled those seen in human NAFLD. The analyses from the aforementioned data obtained from the fructose-consuming monkeys $(n=17)$ show that the number and diameter of the lipid droplets increased with longer duration of consuming the fructose-containing diet. The steatosis score, or number of droplets, also had a significant positive relationship with hepatic fibrosis. In summary, in monkeys, a diet high in fructose causes pathological features in the liver similar to that found in NAFLD in humans.

\section{HUMAN STUDIES}

Hochuli $e t a l^{24}$ conducted a study on 34 normal-weight men in a randomised crossover study. Four isocaloric 3-week interventions with sugar-sweetened beverages (SSB) were performed in random order: medium fructose (MF; $40 \mathrm{~g} / \mathrm{dL}$ ), high fructose (HF; $80 \mathrm{~g} / \mathrm{dL}$ ), high sucrose (HS; $80 \mathrm{~g} / \mathrm{dL}$ ) and high glucose (HG; $80 \mathrm{~g}$ / dL). After each intervention, fasting total plasma fatty acid composition was measured. The results showed that the relative abundance of palmitate (16:0) and the molar fatty acid ratio of palmitate to linoleic acid (16:018:2) as markers of fatty acid synthesis were increased after HF (relative abundance of palmitate: $22.97 \%$ and $5.51 \%$ (percentage of total fatty acids by weight)) and MF (26.1\% and $1.7 \%$, respectively) compared with HS $(19.40 \%-2.91 \%, \mathrm{p}<0.001)$, HG $(19.43 \%-3.12 \%, \mathrm{p}<0.001)$ or baseline $(19.40 \%-2.79 \%, \mathrm{p}<0.001)$. The study showed that even moderate amounts of fructose increase fatty acid synthesis (palmitate) and fasting long-chain acylcarnitines, indicating an impaired fatty acid flux into the $\beta$-oxidation pathway (suggesting reduced fatty acid oxidation in the liver).

A study ${ }^{25}$ found that fructose is a risk factor for the development and progression of NAFLD based on the degree of ATP depletion. Fructose phosphorylation in the liver consumes ATP; consequently, the accumulated ADP serves as substrate for uric acid (UA) formation. These events facilitate hepatic oxidative damage and lipid peroxidation. The study evaluated diabetic adults enrolled in the Action for Health in Diabetes Fatty Liver Ancillary Study ( $\mathrm{n}=244$ ) for whom dietary fructose consumption (estimated by a 130-item food frequency questionnaire) and hepatic ATP (measured by phosphorus magnetic resonance spectroscopy) and UA levels were performed $(n=105)$. In a subset of participants $(n=25)$, an intravenous fructose challenge was used to assess changes in hepatic ATP content. The consumers were stratified as low-fructose $(11.13 \pm 1.33 \mathrm{~g} /$ day $)$ versus high-fructose $(22.3 \pm 1.95 \mathrm{~g} /$ day $)$ consumers. High dietary fructose consumers had slightly lower baseline hepatic
ATP levels and a greater absolute change in hepatic $\alpha$-ATP/inorganic phosphate $(\mathrm{Pi})$ ratio $(0.08$ vs 0.03 ; $\mathrm{p}=0.05)$ and $\gamma$-ATP $/$ Pi ratio after an intravenous fructose challenge ( 0.03 vs $0.06 ; \mathrm{p}=0.06)$. This suggests that a diet high in fructose depletes hepatic ATP levels.

The association between habitual consumption of HFr diets and susceptibility to hepatic ATP depletion after an acute fructose challenge may be due to a compensatory upregulation of fructose-metabolising enzymes in high-fructose consumers. Hydrolysis of ATP during fructose metabolism generates ADP and AMP. The latter is either rephosphorylated by AMP kinase to regenerate ATP, or further degraded to adenosine and ultimately to UA. UA tends to accumulate when the rate of ATP hydrolysis outpaces its regeneration. These studies suggest that the effects of fructose to upregulate its enzymes could lead to a greater ATP depletion and hyperuricaemia in response to fructose. In turn, a more severe ATP depletion could be a mechanism for potentiating liver cell injury in subjects with NAFLD.

Another study ${ }^{26}$ found that replacing SSB with artificially sweetened (calorie-free) beverages (ASB) decreases intrahepatocellular lipid (IHCL) concentrations in overweight subjects with high SSB consumption. Thirty-one healthy subjects with body mass index (BMI) greater than $25 \mathrm{~kg} / \mathrm{m}^{2}$ and a daily consumption of at least $660 \mathrm{~mL}$ ( $22 \mathrm{oz}) \mathrm{SSB}$ were randomised to a 12-week intervention. IHCL was reduced to $74 \%$ of the initial values with ASB $(\mathrm{n}=14 ; \mathrm{p}<0.05)$ but did not change with control. The decrease in IHCL attained with ASB was more important in subjects with IHCL greater than $60 \mathrm{mmol} / \mathrm{L}$ than in subjects with low IHCL. The study concluded that replacing SSB with ASB decreased intrahepatic fat over a 12-week period.

Jin $e t a l^{27}$ found that children with NAFLD are more sensitive to the adverse metabolic effects of fructose beverages than children without NAFLD. A 2-day crossover study was performed in 9 children with NAFLD and 10 matched controls without NAFLD. Plasma lipid levels over two non-consecutive, randomly assigned, 24-hour periods under isocaloric, isonitrogenous conditions with three macronutrient-balanced, consecutive meals and either a fructose-sweetened beverage (FB), or a glucose beverage (GB) being consumed with each meal were measured. The outcomes (plasma glucose, insulin, TG, apoB, high-density lipoprotein cholesterol and non-esterified free fatty acid (FFA) levels) were assessed using mixed models and 24-hour incremental areas under the time-concentration curve. After FB, TG incremental area under the curve was higher versus GB both in children with NAFLD $(p=0.011)$ and those without NAFLD $(p=0.027)$. Moreover, incremental response to FB was greater in children with NAFLD than those without NAFLD $(p=0.019)$. For all subjects, high-density lipoprotein cholesterol declined in the postprandial and overnight hours with $\mathrm{FB}$, but not with $\mathrm{GB}(\mathrm{p}=0.0006)$. Non-esterified fatty acids were not impacted by sugar but were significantly higher in NAFLD. The study concluded 
that children with NAFLD demonstrate an increased sensitivity to the impact of dietary fructose.

A cross-sectional study ${ }^{28}$ supports the role for DNL in the pathogenesis of NAFLD. Sixteen subjects $\left(B M I\left(\mathrm{~kg} / \mathrm{m}^{2}\right)\right.$ : $30.6 \pm 1.2)$ were placed on a hypercaloric diet $(>1000 \mathrm{kcal}$ simple carbohydrates/day, consisting of candy, pineapple juice, sugar-sweetened soft drinks, and/or carbohydrate-loading drink) for 3 weeks and, thereafter, on a hypocaloric diet for 6 months. Since the PNPLA3 genotype may influence the response of serum TGs to stimulation of DNL, the study subjects were genotyped with respect to rs738409 of the PNPLA3 gene and recruited subjects with the PNPLA3-148MM or the PNPLA3-148II homozygous genotype. Metabolic variables and liver fat (measured by proton magnetic resonance spectroscopy) were measured, before and after overfeeding and after hypocaloric diet. The ratio of palmitate $(16: 0)$ to linoleate (18:2n-6) in serum and VLDL TGs was used as an index of DNL. Carbohydrate overfeeding increased weight by $2 \%(1.8 \pm 0.3 \mathrm{~kg} ; \mathrm{p}<0.0001)$ and liver fat by $27 \%$ from $9.2 \pm 1.9 \%$ to $11.7 \pm 1.9 \% \quad(p=0.005)$. DNL increased in proportion to the increase in liver fat and serum TGs in subjects with PNPLA3-148II but not PNPLA3-148MM. During the hypocaloric diet, the subjects lost $4 \%$ of their weight $(3.2 \pm 0.6 \mathrm{~kg} ; \mathrm{p}<0.0001)$ and $25 \%$ of their liver fat content (from $11.7 \pm 1.9 \%$ to $8.8 \pm 1.8 \%$; $\mathrm{p}<0.05$ ). The study concluded that carbohydrate overfeeding for 3 weeks induced a $>10$-fold greater relative change in liver fat $(27 \%)$ than in body weight $(2 \%)$. These data indicate that liver fat avidly accumulates during carbohydrate overfeeding and supports a role for DNL in the pathogenesis of NAFLD.

Schwarz $e t a l^{11}$ performed a study on eight healthy men for 9 consecutive days where they compared the effects of an HFr diet (25\% of energy content) to an isocaloric diet with the same macronutrient distribution but in which fructose was substituted with complex carbohydrate (CCHO). Unlike previous similar studies, neutral energy balance was maintained, hence weight remained stable throughout the study. Stable isotope tracer revealed that the HFr diet was associated with higher DNL (average $18.6 \pm 1.4 \%$ vs $11.0 \pm 1.4 \%$ for CCHO, $\mathrm{p}=0.001)$. Similarly, higher liver fat (median $137 \%$ of CCHO, $\mathrm{p}=0.016$ ) was measured by magnetic resonance spectroscopy in all participants. This study indicates that short-term high-fructose intake is associated with increased DNL and liver fat accumulation in healthy men fed weight-maintaining diets.

A meta-analysis ${ }^{29}$ investigated relevant trials that involved a follow-up of $\geq 7$ days on the effect of fructose on markers of NAFLD. Results were from 13 trials in 260 healthy participants. Seven trials were isocaloric replacement, in which fructose was exchanged isocalorically for other carbohydrates, and six hypercaloric trials, in which the diet was supplemented with excess energy $(+21 \%-$ $35 \%$ energy) from high-dose fructose $(+104-220 \mathrm{~g} /$ day $)$. Although there was no effect of fructose in isocaloric trials, fructose in hypercaloric trials increased both intrahepatic cholestasis (IHCL) $(\mathrm{SMD}=0.45(95 \% \mathrm{CI} 0.18,0.72))$ and alanine transaminase (ALT) $\quad(\mathrm{MD}=4.94 \mathrm{U} / \mathrm{L} \quad(95 \% \mathrm{CI}$ $0.03,9.85))$. The limitations in the study were the inclusion of small trials with short duration ( $\leq 4$ weeks) and energy as a confounding factor (although consuming added fructose seems to drive increased energy intake). ${ }^{30}$ The study concluded that short-term isocaloric exchange of fructose for other carbohydrates does not induce NAFLD changes in healthy partipants however hypercaloric caloric trials increased both IHCL and ALT.

Abdelmalek et $a \hat{l}^{31}$ studied 341 adults enrolled in the NASH Clinical Research Network for whom Block food questionnaire data were collected within 3 months of a liver biopsy. Fructose consumption was estimated based on reporting (frequency×amount) of Kool-Aid, fruit juices and non-dietary soda intake, expressed as servings per week, and classified into none, occasional $(<7$ servings/week) and daily ( $\geq 7$ servings/week). The association of fructose intake with metabolic and histological features of NAFLD was analysed using multiple linear and logistic regression analyses with and without controlling for other confounding factors. After controlling for age, gender, BMI and total calorie intake, daily fructose consumption was associated with lower steatosis grade and higher fibrosis stage $(\mathrm{p}<0.05$ for each). In older adults (age $>48$ years), daily fructose consumption was associated with increased hepatic inflammation $(\mathrm{p}<0.05)$ and hepatocyte ballooning $(p=0.05)$. The study concluded that in patients with NAFLD, daily fructose ingestion is associated with reduced hepatic steatosis but increased fibrosis.

Another study ${ }^{32}$ demonstrated that dietary fructose reduction improves markers of cardiovascular disease risk in patients with NAFLD. This was a 4-week, doubleblinded, randomised, controlled intervention study on overweight (BMI z-score $\geq 85$ th percentile) adolescents. Eligibility criteria included self-identification as Hispanic, ages $11-18$ years; $\mathrm{BMI} \geq 85$ th percentile for age and gender; and average self-reported consumption of at least three servings of sweet beverages (equivalent to 24 fluid oz/day). Sweet beverages were defined as drinks sweetened with added sugars (eg, sodas, sweet tea, sport drinks, flavoured drinks) or naturally sweet beverages (eg, $100 \%$ fruit juice), but did not include artificially sweetened drinks. After the 4-week intervention, adolescents receiving study-provided GB (considered as fructose reduction) had significant improvement in plasma high-sensitivity C-reactive protein (hs-CRP) $(\mathrm{p}=0.028)$, adipose insulin resistance (IR) index $(\mathrm{p}=0.004)$ and plasma FFA $(p=0.027)$ as compared with baseline. In addition, they had a significant reduction in circulating oxidised low-density lipoprotein (oxLDL) levels $(\mathrm{p}=0.034)$. The susceptibility of low-density lipoprotein (LDL) to $\mathrm{Cu}^{2+}$-induced oxidative modification was significantly improved as demonstrated by a twofold increase in LDL lag time $(p=0.010)$. In the group receiving fructose beverages (considered as fructose continuation), there were no significant changes in plasma FFA, hs-CRP, adipose IR index, oxLDL levels 
or the ex vivo oxidative susceptibility of LDL. For both groups, no significant changes were observed in body weight, hepatic fat, liver enzymes, fasting TG and PAI-1 levels after 4 weeks. The study concluded that reducing dietary fructose may be an effective intervention to blunt atherosclerosis progression among patients with NAFLD.

Ma $e t a l^{33}$ observed fatty liver disease using liver attenuation measurements generated from CT in 2634 participants. ALT concentration, a crude marker of fatty liver disease, was measured in 5908 participants. SSB and diet soda intake was estimated using a food frequency questionnaire. Participants were categorised as either non-consumers or consumers (three categories: 1 serving/month to $<1$ serving/week, 1 serving/week to $<1$ serving/day, and $>1$ serving/day) of SSB or diet soda. After adjustment for age, sex, smoking status, Framingham cohort, energy intake, alcohol, dietary fibre, fat (\% energy), protein ( $\%$ energy), diet soda intake and BMI, the ORs of fatty liver disease were $1,1.16(0.88,1.54), 1.32(0.93,1.86)$ and $1.61(1.04,2.49)$ across SSB consumption categories ( $p$ trend $=0.04)$. SSB consumption was also positively associated with ALT levels ( $\mathrm{p}$ trend=0.007). In conclusion, regular SSB consumption was associated with greater risk of fatty liver disease, particularly in overweight and obese individuals. Because NAFLD is an independent risk factor for coronary heart disease, ${ }^{34}$ reducing added dietary fructose may also improve long-term cardiovascular health.

\section{CONCLUSION}

Based on the above evidence, dietary added fructose intake (as found in sucrose or high-fructose corn syrup) when consumed in excess is likely a principal driver of NAFLD and its consequences. Considering that the consumption of added sugars has increased from just $4 \mathrm{lb}$ per person per year in the 1700 s, to approximately $120 \mathrm{lb}$ per person per year, added fructose-induced NAFLD should be considered a public health crisis. Reducing the intake of added fructose should be recommended to prevent and treat patients with NAFLD.

Contributors JJD conceived the paper, drafted the main arguments and performed the literature review. AMS helped with the literature review and drafted the initial manuscript. JJD and JOK provided additional edits to the final manuscript.

Competing interests JJD is the author of the forthcoming book The Salt Fix. Patient consent None.

Provenance and peer review Not commissioned; externally peer reviewed.

Data sharing statement There are no additional data available for this paper.

Open Access This is an Open Access article distributed in accordance with the Creative Commons Attribution Non Commercial (CC BY-NC 4.0) license, which permits others to distribute, remix, adapt, build upon this work non-commercially, and license their derivative works on different terms, provided the original work is properly cited and the use is non-commercial. See: http://creativecommons.org/ licenses/by-nc/4.0/

(C) Article author(s) (or their employer(s) unless otherwise stated in the text of the article) 2017. All rights reserved. No commercial use is permitted unless otherwise expressly granted.

\section{REFERENCES}

1. Szczepaniak LS, Nurenberg P, Leonard D, et al. Magnetic resonance spectroscopy to measure hepatic triglyceride content: prevalence of hepatic steatosis in the general population. Am J Physiol Endocrinol Metab 2005;288:E462-E468.

2. Younossi ZM, Stepanova M, Afendy M, et al. Changes in the prevalence of the most common causes of chronic liver diseases in the United States from 1988 to 2008. Clin Gastroenterol Hepatol 2011;9:524-30.

3. Lim JS, Mietus-Snyder M, Valente A, et al. The role of fructose in the pathogenesis of NAFLD and the metabolic syndrome. Nat Rev Gastroenterol Hepatol 2010;7:251-64.

4. Te Morenga L, Mallard S, Mann J. Dietary sugars and body weight: systematic review and meta-analyses of randomised controlled trials and cohort studies. BMJ 2012;346:e7492.

5. Lazo M, Hernaez R, Eberhardt MS, et al. Prevalence of nonalcoholic fatty liver disease in the United States: the third national health and nutrition examination survey, 1988-1994. Am J Epidemiol 2013;178:38-45.

6. Sun SZ, Empie MW. Fructose metabolism in humans - what isotopic tracer studies tell us. Nutr Metab 2012;9:89.

7. Suzuki T, Douard V, Mochizuki K, et al. Diet-induced epigenetic regulation in vivo of the intestinal fructose transporter Glut5 during development of rat small intestine. Biochem J 2011;435:43-53.

8. Basaranoglu M, Basaranoglu G, Bugianesi E. Carbohydrate intake and nonalcoholic fatty liver disease: fructose as a weapon of mass destruction. Hepatobiliary Surg Nutr 2015;4:109-16.

9. Dekker MJ, Su Q, Baker C, et al. Fructose: a highly lipogenic nutrient implicated in insulin resistance, hepatic steatosis, and the metabolic syndrome. Am J Physiol Endocrinol Metab 2010;299:E685-E694.

10. Chung M, Ma J, Patel K, et al. Fructose, high-fructose corn syrup, sucrose, and nonalcoholic fatty liver disease or indexes of liver health: a systematic review and meta-analysis. Am J Clin Nutr 2014;100:833-49.

11. Schwarz JM, Noworolski SM, Wen MJ, et al. Effect of a high-fructose weight-maintaining diet on lipogenesis and liver fat. J Clin Endocrinol Metab 2015;100:2434-42.

12. Hua X, Yokoyama C, Wu J, et al. SREBP-2, a second basic-helixloop-helix-leucine zipper protein that stimulates transcription by binding to a sterol regulatory element. Proc Natl Acad Sci U S A 1993;90:11603-7.

13. Yokoyama C, Wang X, Briggs MR, et al. SREBP-1, a basic-helixloop-helix-leucine zipper protein that controls transcription of the low density lipoprotein receptor gene. Cell 1993;75:187-97.

14. Olofsson SO, Borèn J. Apolipoprotein B: a clinically important apolipoprotein which assembles atherogenic lipoproteins and promotes the development of atherosclerosis. J Intern Med 2005;258:395-410.

15. Su Q, Tsai J, Xu E, et al. Apolipoprotein B100 acts as a molecular link between lipid-induced endoplasmic reticulum stress and hepatic insulin resistance. Hepatology 2009;50:77-84.

16. Kammoun HL, Chabanon H, Hainault I, et al. GRP78 expression inhibits insulin and ER stress-induced SREBP-1c activation and reduces hepatic steatosis in mice. J Clin Invest 2009;119:1201-15.

17. Rodríguez-Calvo R, Barroso E, Serrano L, et al. Atorvastatin prevents carbohydrate response element binding protein activation in the fructose-fed rat by activating protein kinase A. Hepatology 2009;49:106-15.

18. Roncal-Jimenez CA, Lanaspa MA, Rivard CJ, et al. Sucrose induces fatty liver and pancreatic inflammation in male breeder rats independent of excess energy intake. Metabolism 2011;60:1259-70.

19. Chong MF, Fielding BA, Frayn KN. Mechanisms for the acute effect of fructose on postprandial lipemia. Am J Clin Nutr 2007;85:1511-20.

20. Yamamoto M, Yamamoto I, Tanaka Y, et al. Fatty acid metabolism and lipid secretion by perfused livers from rats fed laboratory stock and sucrose-rich diets. J Lipid Res 1987;28:1156-65.

21. Ackerman Z, Oron-Herman M, Grozovski M, et al. Fructose-induced fatty liver disease: hepatic effects of blood pressure and plasma triglyceride reduction. Hypertension 2005;45:1012-8.

22. Armutcu F, Coskun $O$, Gürel $A$, et al. Thymosin alpha 1 attenuates lipid peroxidation and improves fructose-induced steatohepatitis in rats. Clin Biochem 2005;38:540-7.

23. Cydylo MA, Davis AT, Kavanagh K. Fatty liver promotes fibrosis in monkeys consuming high fructose. Obesity 2017;25:290-3.

24. Hochuli M, Aeberli I, Weiss A, et al. Sugar-sweetened beverages with moderate amounts of fructose, but not sucrose, induce fatty acid synthesis in healthy young men: a randomized crossover study. $J$ Clin Endocrinol Metab 2014;99:2164-72.

25. Abdelmalek MF, Lazo M, Horska A, et al. Higher dietary fructose is associated with impaired hepatic adenosine triphosphate 
homeostasis in obese individuals with type 2 diabetes. Hepatology 2012;56:952-60.

26. Campos V, Despland C, Brandejsky V, et al. Sugar- and artificially sweetened beverages and intrahepatic fat: a randomized controlled trial. Obesity 2015;23:2335-9.

27. Jin R, Le NA, Liu S, et al. Children with NAFLD are more sensitive to the adverse metabolic effects of fructose beverages than children without NAFLD. J Clin Endocrinol Metab 2012;97:E108 8-E1098.

28. Sevastianova K, Santos A, Kotronen A, et al. Effect of short-term carbohydrate overfeeding and long-term weight loss on liver fat in overweight humans. Am J Clin Nutr 2012;96:727-34.

29. Chiu S, Sievenpiper JL, de Souza RJ, et al. Effect of fructose on markers of non-alcoholic fatty liver disease (NAFLD): a systematic review and meta-analysis of controlled feeding trials. Eur J Clin Nutr 2014;68:416-23.
30. DiNicolantonio JJ, O'Keefe JH, Lucan SC. In reply-fructose as a driver of diabetes: an incomplete view of the evidence. Mayo Clin Proc 2015;90:988-90.

31. Abdelmalek MF, Suzuki A, Guy C, et al. Increased fructose consumption is associated with fibrosis severity in patients with nonalcoholic fatty liver disease. Hepatology 2010;51:1961-71.

32. Jin R, Welsh JA, Le NA, et al. Dietary fructose reduction improves markers of cardiovascular disease risk in Hispanic-American adolescents with NAFLD. Nutrients 2014;6:3187-201.

33. Ma J, Fox CS, Jacques PF, et al. Sugar-sweetened beverage, diet soda, and fatty liver disease in the framingham heart study cohorts. $J$ Hepatol 2015;63:462-9.

34. Chhabra R, O'Keefe JH, Patil $\mathrm{H}$, et al. Association of coronary artery calcification with hepatic steatosis in asymptomatic individuals. Mayo Clin Proc 2013;88:1259-65. 